\title{
HOW INSPIRING IS YOUR APP? A USABILITY TAKE ON AN APP FOR ASTHMA MEDICATION ADHERENCE
}

\author{
Ana Ferreira ${ }^{1,2}$, Rute Almeida ${ }^{1,2}$, Cristina Jácome ${ }^{1,2}$, José Fernandes ${ }^{4}$, João A. Fonseca ${ }^{1,2,3}$ \\ and Pedro Vieira-Marques ${ }^{1,2}$ \\ ${ }^{1}$ CINTESIS - Center for Health Technology and Services Research \\ ${ }^{2}$ Departamento Medicina da Comunidade, Informação e Decisão em Saúde (MEDCIDS) \\ Faculty of Medicine, University of Porto, Portugal \\ ${ }^{3}$ Medicina, Educação, I\&D e Avaliação, Lda (MEDIDA), Porto, Portugal \\ ${ }^{4}$ Bloomidea, Braga, Portugal
}

\begin{abstract}
The low level of awareness and usability of existing mobile health (mHealth) applications still hinders people, especially patients, to take advantage of such applications to improve their daily lives and health outcomes. This is also true for patients with asthma however, the use of smartphones and gamification, can be a solution to change behaviours regarding inhaled medication adherence and to improve asthma monitoring closer to the patients' needs. This paper presents a usability study of InspirerMundi, an mHealth gamified app developed to include various monitoring functionalities to improve inhaled medication adherence in adolescents and adults with asthma. A well-defined protocol with specific tasks was applied to 8 participants, who never used the app before nor had asthma (4 female and 4 iOS users), and results show that it was possible to find the most pressing issues regarding usability, right from the beginning. This work helps to realize that mHealth applications need to be tested for usability and functionalities adequacy with a varied sample closely related to technology and personal characteristics (e.g., type of smartphone, gender, age, mobile game-oriented), for which those applications need to be prepared.
\end{abstract}

\section{KEYWORDS}

Usability, Mobile Health Application, Asthma Medication Adherence

\section{INTRODUCTION}

The rapid growth of mobile health (mHealth) applications (apps) has allowed new ways to improve medication control, monitorization and health promotion. However, there is still a lot to be done regarding the level of awareness and usability of such apps (Kayyali et al., 2017) to make sure that: a) patients know more/better ways to treat their condition; and b) apps do not go into the market without being tried for their majority of problems (Boulos et al., 2014). Especially in the healthcare domain, effects of using mobile apps can be crucial as the lack of standardization and adequate scientific validation can cause serious problems to the user's physical and mental health (Parati et al., 2017). In terms of respiratory diseases (e.g., asthma), patients' low adherence to inhaled medication is one of the main factors that can hinder the clinical improvement and maintenance of patients' quality of life (Morton et al., 2014) (van Dulmen et al., 2007). Applications that can help this to be concretized are critical. The use of smartphones and gamification approaches can be relevant options to consider for changing behaviours to improve asthma medication adherence closer to the patients' needs (Haase et a., 2017) (Marcano et al., 2013) (Morton et al., 2017).

With all this in mind, the mHealth app InspirerMundi was developed with various monitoring and gamification functionalities aiming to improve inhaled medication adherence in adolescents and adults with asthma (Jácome et al., 2017). This paper presents a first round of usability tests that were performed on the InspirerMundi app in order to identify: functionalities that are hard to execute, lack of clarity in language and design as well as the average time tasks take to complete, to identify areas that need improvement. 


\section{METHODS}

\subsection{Sampling}

This was a cross-sectional study. The participants were recruited using a convenience sampling procedure and were included if they were over 18 years old, frequently used a smartphone, did not have asthma and had never used the InspirerMundi app before. The focus was to have a balanced sample regarding gender and the use of different operating systems users (e.g., iOS and Android). The sample integrated non-asthmatic people to avoid previous users experience both with such apps and different type of inhaled medication as well as any other type of emotional and behavioural involvement.

\subsection{Protocol and Materials}

The study protocol comprised 4 steps: 1) researchers presented the study and its objectives to the participants by providing the same information they would find when downloading the app from the online stores ${ }^{1}$; 2) participants read and signed an information consent describing the main goal of the study and how collected data would be processed and analysed; 3) participants performed nine pre-defined tasks (Section 2.3) with smartphones provided by the research team, which were video recorded with a web cam and Mr. Tappy support (Figure 1); and, at the end, 4) participants filled a usability and satisfaction questionnaire.
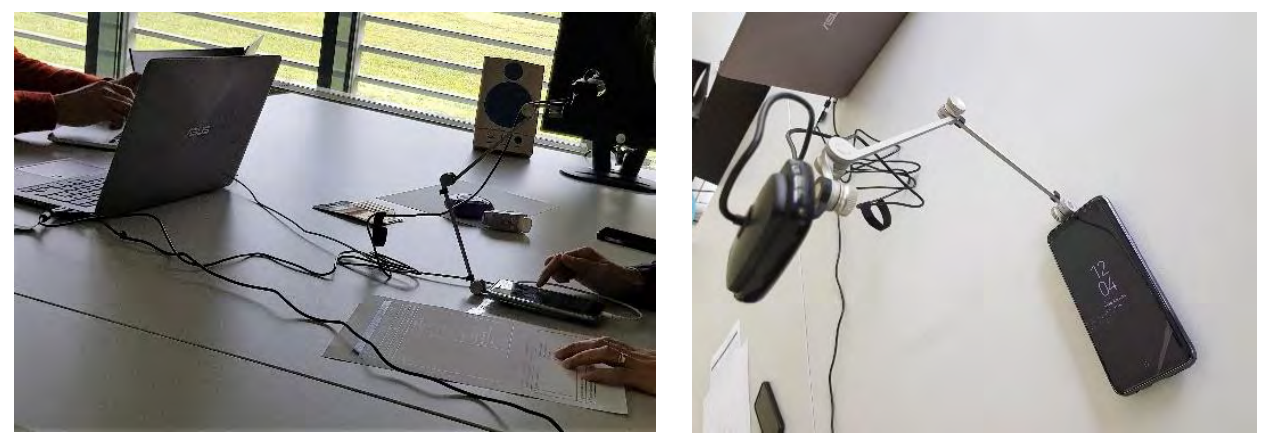

Figure 1. Setup of the video recording of the participants' performance and smartphone display

\subsection{Performed Tasks and Survey}

The nine tasks focused on activities to be daily executed by patients who want to improve their adherence to asthma medication. These were: 1) login with anonymous credentials; 2) answer the Control of Allergic Rhinitis and Asthma Test (CARAT) (Fonseca et al., 2010); 3) answer a weekly symptoms survey; 4) insert two inhaled medications and one pill medication; 5) register the taking of the three previously inserted medications; 6) modify the posology of one of the inserted medications; 7) register an SOS medication; 8) make an Inspirer request; and 9) check the statistics for the medication adherence. Every task was completed by two experts (one using Android, the other iOS), who knew the application, to create a baseline for each task duration. During the study, no help was provided to the participants when performing the tasks. There were two observers in the room and the participants could change the sequence of the tasks' execution and give up or stop whenever they thought fit, without any intervention or suggestion from the observers.

An anonymous post-study survey was given to the participants and consisted of four parts: i. demographic data; ii. use of smartphones; iii. satisfaction and usability of the app; and iv. security and privacy issues.

${ }^{1}$ https://play.google.com/store/apps/details?id=com.bloomidea.inspirers\&hl=en or https://itunes.apple.com/pt/app/inspirermundi/id1441138269?mt=8 


\section{RESULTS}

\subsection{Participants}

The sample comprised eight participants (a number that helps to initially detect between 50 to 80 percent of the problems the app may have (Ritch, 2009)) with an average of 36 (28-44) years old, 50\% were female, $50 \%$ were Android users and all of them had a high education degree and frequently use a smartphone.

\subsection{Tasks Scores}

Most tasks were successfully performed by all the participants ( 7 out of 9), however, one of the most relevant ones (Task 5) had two failures and 4 partial successes. This task implied the registration of a video image of the taken medication and only when that medication was in the format of a pill case did the participants understood that they were supposed to use the smartphone camera to make a short video/photograph. Another issue concerned Task 6, where it was not obvious to the participants how this could be achieved since the functionality that allows to do it was "hidden" and not close to the available list of medications. Figure 2 shows the variability in time needed to complete a task when comparing the users and the experts' performance. Tasks 5, 6 and 7 are the highest duration tasks involving more interactions with the app from the participants, probably for the reasons already presented.

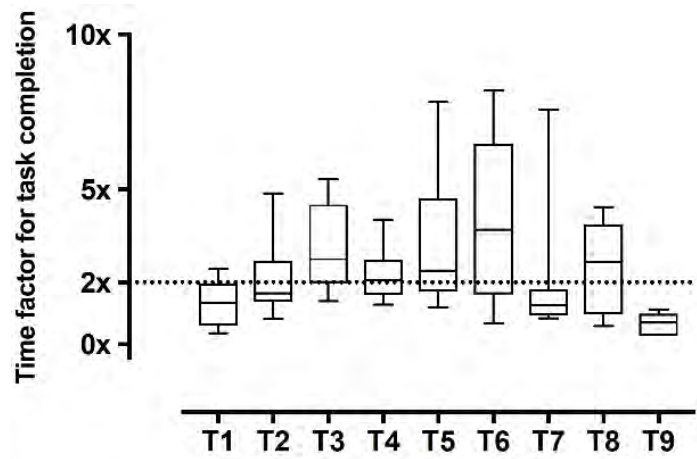

Figure 2. Time factor for task completion (experts vs participants) (T5 was finished by 6 participants and T6 by 5)

\subsection{Post-Study Survey}

Most participants use smartphones mainly to: a) access the Internet (more Female and Android users); b) use mobile apps (more Male and Android users); c) check social networks (more Female and IOS users as well as non-gamers); and d) to take pictures (more Female and IOS users as well as non-gamers).

Since the evaluated app focus on gamification elements to promote adherence to medication, the main usability aspects that need improvement are detailed for gamers and non-gamers in Table 1. Despite some differences, both types of users agreed that learning and application usage and app interactions were critical to improve. Regarding the security issues, some participants avoid giving similar apps access to the stored contacts and photos (more Female, IOS and non-gamers users) while all the participants had no problems in allowing those apps accessing their location as well as sending them notifications. 
Table 1. Critics of usability characteristics depending on the type of participant (mobile gamer or not)

\begin{tabular}{|c|c|c|c|c|}
\hline \multirow[b]{2}{*}{ Usability characteristics } & \multicolumn{2}{|c|}{$\begin{array}{l}\text { Never plays games } \\
\text { in smartphone }\end{array}$} & \multicolumn{2}{|c|}{$\begin{array}{c}\text { Regularly plays } \\
\text { mobile games }\end{array}$} \\
\hline & $\begin{array}{l}\text { More } \\
\text { critical }\end{array}$ & $\begin{array}{c}\text { Less } \\
\text { critical }\end{array}$ & $\begin{array}{l}\text { More } \\
\text { critical }\end{array}$ & $\begin{array}{c}\text { Less } \\
\text { critical }\end{array}$ \\
\hline Size and disposition of buttons/icon/menus/content are appropriate & & $\mathrm{X}$ & $\mathrm{X}$ & \\
\hline Quality of buttons/icon/menus/content & & & & $\mathrm{X}$ \\
\hline Functionalities, menu and button components are efficient & & & $\mathrm{X}$ & \\
\hline Learning and application usage is fast & $\mathrm{X}$ & & $\mathrm{X}$ & \\
\hline Language used in instructions and menus/icons is clear & & & & $\mathrm{X}$ \\
\hline Display changing is correct/logical/appropriate and without interruptions & $\mathrm{X}$ & & & $\mathrm{X}$ \\
\hline Interactions are consistent and intuitive & $\mathrm{X}$ & & $\mathrm{X}$ & \\
\hline App is fun to use and includes interesting gaming components & & $\mathrm{X}$ & $\mathrm{X}$ & \\
\hline
\end{tabular}

\section{DISCUSSION AND LESSONS LEARNED}

In terms of usability of the mHealth InspirerMundi app, this small study already gave important clues on what were the main issues to solve regarding two of the main features. One of the core features, the recognition of the inhaled medication, was not that straightforward to grasp and so needed attention. Besides being a common gesture in mobile app usage (taking a photo or making a video) the subject being captured is not. The inclusion of an explanatory description with images/video before the actual process takes place will allow for the first-time users to better understand what is expected. This shows how important these types of studies are before releasing the apps and how they can help to identify a lot more issues than anticipated. Another important aspect is the fact that the study was applied to a sample population completely alienated from the theme at hand which, besides avoiding some cognitive or behavioural bias, made us have more confidence in the obtained results as there was no pressure for those participants to do well on that specific app. To also notice that an average expert baseline time for the performed tasks helps comparing times and efficiency on a normal daily usage. In some tasks the users took on average between 1,5 to 2 times more to complete when compared with experts, this situation is natural as the interface is new to the users and is expected that after the initial steps the difference will decrease. However, this can also be a good indicator regarding what aspects of the app need to be improved to prevent frustration and dropout on the initial stages of its use. Finally, although there is the necessity to focus on the basic functionalities first, allowing the removal of obstacles that may hinder the correct assessment of the app's effective purpose, there is the necessity of assessing gamification components, that will need, nonetheless, a more extensive period of testing.

For the satisfaction, perception and attitudes of study participants towards the app, it was clear that the majority of users were satisfied with most functionalities however, different attitudes arose for mobile gamers. Non-gamers focus more on the consistency and easiness of actions, while gamers also focus on those characteristics, plus on the aesthetics aspect such as size and disposition of graphical components and how useful and fun they are. This was expected, so, if the app is a health serious game, recommendations are to tackle usability issues preferably with a sample integrating both views.

In terms of security issues, although the number of security questions was very small, results show that gender, together with the type of smartphone (e.g., iOS or Android) or mobile game habits, can influence the perception of providing identifiable personal data. This must be further analysed in future research.

Limitations comprise the use of an equipment (e.g., Mr. Tappy) that was not helpful to perform the task of video/photographing an object. Nevertheless, this may only partly explain the problems executing this task, as the equipment was used only with three participants. Also, only healthy adults were included and it was not possible to compare their performance with patients with asthma, who need to use such app on a daily basis in the interest to improve their symptoms and quality of life. Future studies should include participants both healthy and with asthma, from distinct age groups. 


\section{CONCLUSION}

This work helped to understand how mHealth apps, integrating gamification, need to be assessed not only for the basic functionalities, but also to the adequacy of each individual user. In spite that participants were not patients with the condition addressed by the app, they can have other relevant characteristics to be taken in consideration (e.g., gender, age, type of smartphone, game-oriented) and for which this type of apps need to be prepared. Future work includes performing a longer study with patients with asthma, from adolescents to older adults, in order to better adequate and personalize the app according to their lifestyles and needs.

\section{ACKNOWLEDGEMENT}

This work was financed by ERDF (European Regional Development Fund ) funds through the COMPETE 2020 - Operacional Programme for Competitiveness and Internationalisation (POCI), and by Portuguese funds through FCT - Fundação para a Ciência e a Tecnologia in the framework of the project POCI-01-0145-FEDER-029130 ("mINSPIRERS-mHealth para medição e melhoria da adesão à medicação nas doenças respiratórias obstrutivas crónicas - generalização e avaliação de tecnologias de gamificação, suporte por pares e processamento avançado de imagem").

It was also supported by FCT through the Project TagUBig - Taming Your Big Data (IF/00693/2015) from Researcher FCT Program funded by National Funds through FCT - Fundação para a Ciência e a Tecnologia. In addition, Cristina Jácome has a post-doctoral grant (SFRH/BPD/115169/2016) funded by FCT, co-financed by the European Social Fund $(\mathrm{POCH})$ and Portuguese national funds from MCTES (Ministério da Ciência, Tecnologia e Ensino Superior).

\section{REFERENCES}

Boulos, M. N., Brewer, A. C., Karimkhani, C., Buller, D. B., \& Dellavalle, R. P. (2014). Mobile medical and health apps: state of the art, concerns, regulatory control and certification. Online journal of public health informatics, 5(3), 229. doi:10.5210/ojphi.v5i3.4814.

Fonseca JA, Nogueira-Silva L, Morais-Almeida M, Azevedo L, Sa-Sousa A, Branco-Ferreira M, et al. (2010). Validation of a questionnaire (CARAT10) to assess rhinitis and asthma in patients with asthma. Allergy. 65:1042-8.

Haase J, Farris KB, Dorsch MP. (2017). Mobile Applications to Improve Medication Adherence. Telemed J E Health. 23:75-9.

Jácome C, Almeida R, Teixeira J, Vieira-Marques P, Vilaça R, Fernandes J, et al. (2017). Inspirers: An app to measure and improve adherence to inhaled treatment. Proceedings of the International Conference e-Health 2017; Lisboa.135-9.

Kayyali, R., Peletidi, A., Ismail, M., Hashim, Z., Bandeira, P., \& Bonnah, J. (2017). Awareness and Use of mHealth Apps: A Study from England. Pharmacy (Basel, Switzerland), 5(2), 33. doi:10.3390/pharmacy5020033.

Marcano Belisario JS, Huckvale K, Greenfield G, Car J, Gunn LH. (2013). Smartphone and tablet self-management apps for asthma. The Cochrane database of systematic reviews. CD010013.

Morton RW, Elphick HE, Rigby AS, Daw WJ, King DA, Smith LJ, et al. (2017). STAAR: a randomised controlled trial of electronic adherence monitoring with reminder alarms and feedback to improve clinical outcomes for children with asthma. Thorax. 72:347-54.

Morton RW, Everard ML, Elphick HE. (2014). Adherence in childhood asthma: the elephant in the room. Arch Dis Child. 99:949-53.

Parati, G., Torlasco, C., Omboni, S. et al. (2017). Smartphone Applications for Hypertension Management: a Potential Game-Changer That Needs More Control. Curr Hypertens Rep. 19: 48. https://doi.org/10.1007/s11906-017-0743-0.

Ritch Macefield. 2009. How to specify the participant group size for usability studies: a practitioner's guide. J. Usability Studies 5, 1 (November 2009), 34-45.

van Dulmen S, Sluijs E, van Dijk L, de Ridder D, Heerdink R, Bensing J. (2007). Patient adherence to medical treatment: a review of reviews. BMC Health Serv Res. 7:55. 